Working Paper 97-23

Business Economics Series 02

April 1997
Departamento de Economía de la Empresa

Universidad Carlos III de Madrid

Calle Madrid, 126

28903 Getafe (Spain)

Fax (34-1) 624-9608

\title{
EMPIRICAL DISTRIBUTIONS OF STOCK RETURNS: EUROPEAN SECURITIES MARKETS, 1990-95
}

\author{
Felipe Aparicio and Javier Estrada ${ }^{*}$
}

\begin{abstract}
The assumption that daily stock returns are normally distributed has long been disputed by the data. In this article we test (and clearly reject) the normality assumption using time series of daily stock returns for thirteen European securities markets. More importantly, we fit to the data four alternative specifications, find overall support for the scaled-t distribution (and partial support for a mixture of two Normal distributions), and quantify the magnitude of the error that stems from predicting the probability of obtaining returns in specified intervals by using the Normal distribution. We conclude by arguing that normality may be a plausible assumption for monthly (but not for daily) stock returns.
\end{abstract}

Keywords

Time series of stock returns. Nonnormality. Forecasting.

JEL Number: G15

Department of Statistics and Econometrics and Department of Business Economics, Universidad Carlos III de Madrid (Madrid, Spain). EMAILS: <aparicio@est-econ.uc3m.es> and<estrada@eco.uc3m.es>. We would like to thank Chris Adcock, Asani Sarkar, Miguel Sofer, Allan Timmerman, and seminar participants at Gothenburg University for valuable comments. The views expressed below and any errors that may remain are entirely our own. 


\section{I- INTRODUCTION}

The assumption that stock returns are normally distributed is widely used, implicitly or explicitly, in theoretical finance. Investors' preferences can be modeled in a simple way by assuming mean-variance behavior. However, as is well known, this type of behavior is consistent with the more general criterion of expected-utility maximization under either one of two conditions, namely, that investors' utility functions are quadratic or that stock returns are (jointly) normally distributed. Since, as is also well known, a quadratic utility function exhibits some implausible properties, ${ }^{1}$ mean-variance behavior is usually justified through the assumption of normally-distributed stock returns. Therefore, the widespread use of mean-variance behavior, together with the implausibility of quadratic utility functions, may help to explain the popularity of the normality assumption for stock returns. Such popularity is, of course, strengthened by the fact that normally-distributed stock returns are an implication of the random walk theory of stock prices.

From a theoretical point of view, the normality of stock returns is questionable if information does not arrive linearly to the market, or, even if it does, if investors do not react linearly to its arrival. In both cases, a leptokurtic distribution of stock returns should be observed. If information arrives to the market in infrequent clumps instead of in a linear fashion, investors would be forced to react similarly; in other words, if the distribution of information is leptokurtic, so should be the distribution of stock returns. Alternatively, if information arrives to the market linearly but investors ignore it until trends are well in place, and then react in a cumulative fashion to all the information ignored up to that point, a leptokurtic distribution of stock returns would also be obtained; ${ }^{2}$ see Peters (1991). Thus, both arguments suggest that the distribution of stock returns should have fatter tails than expected under the Normal distribution. ${ }^{3}$

\footnotetext{
${ }^{1}$ A plausible utility function should exhibit decreasing absolute risk aversion, constant relative risk aversion, and increasing risk tolerance. However, the quadratic utility function exhibits increasing absolute risk aversion, increasing relative risk aversion, and decreasing risk tolerance.

${ }^{2}$ Note that under this second theory, unlike under the first, changes in stock prices depend on past information, thus contradicting the efficient market hypothesis.

${ }^{3}$ The leptokurtosis in stock returns motivated the proliferation of ARCH-type models, which seek to incorporate the information contained in the tails of a distribution of stock returns into time series models. For a literature review, see Bollerslev, Chou, and Kroner (1992).
} 
Empirical evidence against the normality assumption, on the other hand, has been mounting since the pioneering articles by Mandelbrot (1963), Fama (1965), and Clark (1973). Mandelbrot (1963) argued that price changes can be characterized by a stable Paretian distribution with a characteristic exponent less than 2, thus exhibiting fat tails and an infinite variance. ${ }^{4}$ He directly tested the infinite-variance hypothesis by computing the sample variance of a large number of samples containing the returns of cotton prices, and found that the variances did not converge to any limiting value. Rather, they evolved in an erratic fashion, just as would be expected under the infinite-variance hypothesis.

Fama (1965), using the thirty stocks of the Dow Jones Industrial Average, confirmed Mandelbrot's (1963) hypothesis that a stable Paretian distribution with a characteristic exponent less than 2 describes stock returns better than a Normal distribution. Thus, since stable Paretian markets tend to evolve in jumps (rather than continuously and smoothly like Gaussian markets), he concluded that stocks are riskier than indicated by the standard deviation of a Normal distribution. ${ }^{5}$

The infinite variance of stable Paretian distributions, and the fact that if stock returns follow this distribution then the usual statistical tools may be badly misleading, led many researchers to look for alternatives. Clark (1973) argued in favor of a finite-variance subordinated stochastic process and found that a member of this class (the lognormal distribution) fitted data on cotton futures prices better than a stable Paretian distribution.

More recently, using weekly data for the period 1928-89, Peters (1991) found that the distribution of the S\&P 500 stock returns exhibits negative skewness, fat tails, and a high peak. He also found that the probability of a three-sigma event under the empirical distribution of stock returns is roughly twice as large as the probability that would be expected under a Normal distribution.

We analyze in this article the distributions of stock returns of thirteen European securities markets during the first half of the decade. We start by testing the normality assumption for daily stock returns, which we clearly reject in all markets. Then, we attempt to find the specification that best fits the empirical distribution of stock returns in each

\footnotetext{
${ }^{4}$ When the characteristic exponent of a stable Paretian distribution is exactly equal to 2, then the Normal distribution is obtained. Hence, the latter is a special case of the former.

${ }^{5}$ In fact, in a stable Paretian market, the sample standard deviation cannot be used as a meaningful measure of risk. This is due to the fact that this statistic exhibits a very erratic behavior in the sense that, as the sample size increases, it does not converge to any given value.
} 
market, and find overall support for the scaled-t distribution and partial support for a mixture of two Normal distributions. Then, we quantify the magnitude of the error that stems from predicting the probability of obtaining returns in specified intervals by using the Normal distribution, and find that such specification significantly underestimates the risk of investing in European stocks. Finally, we analyze the distributions of monthly stock returns, and find that they are reasonably-well described by a Normal distribution.

The rest of the article is organized as follows. In section II, we describe the data and run three tests of normality. In section III, we introduce the statistical distributions to be fitted to the data. In section IV, we report and discuss the results of our estimations. In section $\mathrm{V}$, we assess the magnitude of the error that stems from predicting the probability of obtaining returns in specified intervals by assuming a Normal (instead of a more appropriate) distribution. In section VI, we analyze the distributions of monthly stock returns. And, finally, in section VII, we summarize the main findings of our study. An appendix with figures concludes the article.

\section{II- DATA AND TESTS OF NORMALITY}

The sample under consideration consists of thirteen European securities markets, namely, Austria (AUS), Belgium (BEL), Denmark (DEN), England (ENG), Finland (FIN), France (FRA), Germany (GER), Italy (ITA), Netherlands (NET), Norway (NOR), Spain (SPA), Sweden (SWE), and Switzerland (SWI). The behavior of each of these markets is summarized by the Financial Times Actuaries Indices, published daily in the Financial Times. We also analyze the distribution of a World index (WOR), which is computed on the basis of 2,249 stocks worldwide. The sample period extends from January 1, 1990, through December 31, 1994; that is, 1,304 daily data points from the first half of the decade. The temporal behavior of the fourteen indices under consideration is shown in part Al of the appendix.

The series analyzed for each market is the series of returns, where returns are defined as the first difference of the natural logarithm of each index; that is, $R_{t}=100\left[\ln \left(I_{t}\right)-\ln \left(I_{t-1}\right)\right]$, where $R_{t}$ and $I_{t}$ are the return and the index in day $t$, respectively. Table 1 below summarizes some relevant information about the empirical distributions of stock returns under consideration. The statistics reported are the mean, standard deviation, minimum and 
maximum return during the sample period, coefficients of skewness and kurtosis, and standardized coefficients of skewness and kurtosis.

TABLE 1: Sample Moments of the Distributions of Daily Stock Returns

\begin{tabular}{lrrrrrrrr}
\hline Market & Mean & \multicolumn{1}{c}{ SD } & \multicolumn{1}{c}{ Min } & \multicolumn{1}{c}{ Max } & \multicolumn{1}{c}{ Skw } & \multicolumn{1}{c}{ SSkw } & \multicolumn{1}{c}{ Krt } & \multicolumn{1}{c}{ SKrt } \\
\hline AUS & -0.0064 & 1.1853 & -7.5998 & 6.9370 & 0.1758 & 2.5918 & 6.1939 & 45.6561 \\
BEL & -0.0020 & 0.7190 & -5.5734 & 6.9116 & 0.1069 & 1.5761 & 14.6793 & 108.2030 \\
DEN & -0.0030 & 0.8232 & -5.8997 & 4.9312 & -0.0936 & -1.3803 & 5.9536 & 43.8846 \\
ENG & 0.0180 & 0.8185 & -3.9943 & 5.5348 & 0.3340 & 4.9238 & 3.4455 & 25.3974 \\
FIN & 0.0377 & 1.2440 & -5.4757 & 5.2919 & 0.2328 & 3.4316 & 2.1259 & 15.6700 \\
FRA & -0.0026 & 0.9996 & -7.2685 & 5.4874 & -0.2973 & -4.3833 & 3.8782 & 28.5867 \\
GER & 0.0046 & 1.0478 & -10.3649 & 5.2958 & -0.6131 & -9.0381 & 9.7663 & 71.9886 \\
ITA & -0.0017 & 1.3691 & -8.2403 & 5.2754 & -0.1918 & -2.8278 & 2.2403 & 16.5131 \\
NET & 0.0241 & 0.7273 & -3.6398 & 3.0222 & -0.3147 & -4.6391 & 1.6304 & 12.0176 \\
NOR & 0.0069 & 1.3307 & -8.8584 & 10.8018 & 0.3662 & 5.3988 & 8.8477 & 65.2171 \\
SPA & -0.0021 & 1.1249 & -8.6287 & 6.7887 & -0.2627 & -3.8733 & 5.8811 & 43.3505 \\
SWE & 0.0282 & 1.2504 & -6.8453 & 9.3145 & 0.5003 & 7.3755 & 5.7016 & 42.0272 \\
SWI & 0.0306 & 0.9313 & -7.2125 & 5.3787 & -0.6884 & -10.1489 & 7.3683 & 54.3125 \\
WOR & -0.0023 & 0.6535 & -4.2796 & 3.9281 & -0.0142 & -0.2088 & 5.5388 & 40.8272 \\
\hline \hline
\end{tabular}

Sample size $=1,304$ for all markets. Mean returns, standard deviations (SD), minimum returns (Min), and maximum returns (Max) are all expressed in percentages. Skw $=$ Skewness $=m_{3} / s^{3}$ and Krt $=$ Kurtosis $=m_{4} / s^{4}-3$, where $m_{1}$ and $s$ are the $i$ th central sample moment and the sample standard deviation of each distribution, respectively; both coefficients are computed with a finite-sample adjustment. SSkw = Standardized skewness and SKrt $=$ Standardized kurtosis.

Preliminary evidence on the normality of each distribution of stock returns under consideration can be gathered from the last four columns of Table 1; that is, by considering the third and fourth central moments of each distribution. Under the assumption of normality, the coefficients of skewness and excess kurtosis are asymptotically distributed as $N(0,6 / T)$ and $N(0,24 / T)$, respectively, where $T$ is the sample size. Hence, values of these standardized coefficients (SSkw and SKrt) outside the range $[-1.96,1.96]$ indicate, at the 5\% significance level, significant departures from normality.

Table 1 shows that not all the distributions are negatively skewed, as daily data from the U.S. typically shows; this table shows that eight markets display negative skewness and the other six markets display positive skewness. Note, however, that the coefficients of standardized skewness show that the observed asymmetry is not significant in three of the fourteen markets under consideration. In addition, the last column of Table 1 shows that all fourteen distributions are clearly leptokurtic, thus exhibiting fat tails (and high peaks). The departures from normality detected by the coefficients of standardized skewness and kurtosis can also be seen in the histograms displayed in part A2 of the appendix, where Normal distributions generated by the sample mean and standard deviation of each market are shown together with the observed histograms. 
The coefficients of standardized skewness and kurtosis provide strong evidence about departures from normality, but more formal conclusions can be reached through the tests of normality reported below in Table 2 . Although the three tests use different information, ${ }^{6}$ the results of all three point in the same direction, namely, to the outright rejection of the normality assumption. ${ }^{7}$

TABLE 2: Tests of Normality (Daily Stock Returns)

\begin{tabular}{lrrrrrrr}
\hline & \multicolumn{3}{c}{ Goodness of Fit } & \multicolumn{2}{c}{ Kolmogorov-Smimov } & \multicolumn{2}{c}{ Jarque-Bera } \\
\cline { 2 - 8 } Market & Statistic & $\mathrm{df}$ & $p$-value & Statistic & $p$-value & Statistic & $p$-value \\
\hline AUS & 153.704 & 9 & 0.0000 & 0.0748 & $9.2 \mathrm{e}-07$ & $2,091.182$ & 0.0000 \\
BEL & 159.076 & 7 & 0.0000 & 0.0845 & $1.7 \mathrm{e}-08$ & $11,710.330$ & 0.0000 \\
DEN & 118.647 & 7 & 0.0000 & 0.0716 & $3.2 \mathrm{e}-06$ & $1,927.768$ & 0.0000 \\
ENG & 45.830 & 10 & $1.5 \mathrm{e}-06$ & 0.0365 & 0.0621 & 669.261 & 0.0000 \\
FIN & 123.383 & 13 & 0.0000 & 0.0612 & $1.2 \mathrm{e}-04$ & 257.335 & 0.0000 \\
FRA & 43.022 & 9 & $2.1 \mathrm{e}-06$ & 0.0441 & 0.0126 & 836.407 & 0.0000 \\
GER & 75.741 & 7 & $1.0 \mathrm{e}-13$ & 0.0554 & $6.7 \mathrm{e}-04$ & $5,264.041$ & 0.0000 \\
ITA & 85.782 & 13 & $8.9 \mathrm{e}-13$ & 0.0444 & 0.0117 & 280.691 & 0.0000 \\
NET & 64.326 & 13 & $8.7 \mathrm{e}-09$ & 0.0504 & 0.0026 & 165.953 & 0.0000 \\
NOR & 79.617 & 8 & $5.8 \mathrm{e}-14$ & 0.0594 & $2.0 \mathrm{e}-04$ & $4,282.456$ & 0.0000 \\
SPA & 80.346 & 9 & $1.4 \mathrm{e}-13$ & 0.0629 & $6.6 \mathrm{e}-05$ & $1,894.244$ & 0.0000 \\
SWE & 128.317 & 9 & 0.0000 & 0.0626 & $7.3 \mathrm{e}-05$ & $1,820.680$ & 0.0000 \\
SWI & 92.872 & 9 & $4.4 \mathrm{e}-16$ & 0.0561 & $5.5 \mathrm{e}-04$ & $3,052.850$ & 0.0000 \\
WOR & 89.716 & 9 & $1.9 \mathrm{e}-15$ & 0.0629 & $6.6 \mathrm{e}-05$ & $1,666.898$ & 0.0000 \\
\hline \hline
\end{tabular}

The goodness-of-fit test follows a Chi-square distribution with the degrees of freedom (df) indicated above. The asymptotic critical value for the Kolmogorov-Smirnov test at the $5 \%$ significance level is 0.038 . The Jarque-Bera test is asymptotically distributed as a Chi-square with 2 degrees of freedom; its critical value at the $5 \%$ significance level is 5.99 .

The results in Table 2 should come as no surprise; virtually all studies that use daily data also reject the normality of stock returns. In order to test what specification describes the data better than the Normal distribution, we consider in the next part four alternative distributions. We admit from the outset that we have no underlying financial theory to justify the use of all specifications. Rather, our purpose is to fit four distributions that allow for the characteristics of the data discussed above, to determine which one of those distributions best fits each market, and to quantify the error that can be made by predicting the probability of obtaining returns in specified intervals by using the Normal distribution.

\footnotetext{
${ }^{6}$ The Jarque-Bera test uses information on the third and fourth moments of a distribution. The goodness-of-fit test divides a distribution in intervals and compares, across intervals, the observed returns with those that would be expected if the underlying distribution were Normal. Finally, the Kolmogorov-Smirnov test computes the maximum distance between an observed cumulative distribution and the Normal cumulative distribution.

${ }^{7}$ At the $5 \%$ significance level, the null hypothesis of normality is not rejected only in the English market under the Kolmogorov-Smirnov test.
} 


\section{III- ALTERNATIVE DISTRIBUTIONS FOR STOCK RETURNS}

The results reported and discussed above indicate that the fourteen markets we consider are characterized by somewhat skewed distributions with fat tails and high peaks. As a result, we consider in this part three specifications that allow for leptokurtosis and one that also allows for skewness.

The Logistic Distribution. This distribution, which is very similar to the Normal but has thicker tails, was first suggested as appropriate to model stock returns by Smith (1981), and subsequently tested by Gray and French (1990) and Peiró (1994). The density function of the logistic distribution can be written as

$$
f(x)=\frac{\exp \left(\frac{x-\mu}{\alpha}\right)}{\alpha\left[1+\exp \left(\frac{x-\mu}{\alpha}\right)\right]^{2}},
$$

where $\mu(-\infty<\mu<\infty)$ is a location parameter and $\alpha(\infty>0)$ is a dispersion (or scale) parameter. If $R_{t}$ follows a logistic distribution, then $E\left(R_{t}\right)=\mu$ and $\operatorname{Var}\left(R_{t}\right)=\sigma^{2}=\left(\pi^{2} / 3\right) \alpha^{2}$.

The Scaled- $t$ Distribution. Praetz (1972), Blattberg and Gonedes (1974), Gray and French (1990), and Peiró (1994) have reported that this specification fits stock returns better than many competing alternatives. The density function of the scaled- $t$ distribution is given by

$$
f(x)=\frac{\Gamma\left(\frac{v+1}{2}\right)}{\Gamma(v / 2) \sqrt{\pi(v-2) \sigma^{2}}} \cdot\left[1+\frac{(x-\mu)^{2}}{(v-2) \sigma^{2}}\right]-\left(\frac{v+1}{2}\right),
$$

where $\Gamma(\bullet)$ represents the gamma function, $\mu(-\infty<\mu<\infty)$ and $\sigma^{2}\left(\sigma^{2}>0\right)$ represent a location and a dispersion parameter, respectively, and $v(v>0)$ is a degrees of freedom parameter. If $R_{t}$ follows a scaled- $t$ distribution and $v>2$, then $E\left(R_{t}\right)=\mu$ and $\operatorname{Var}\left(R_{t}\right)=\sigma^{2}$.

The Exponential Power Distribution. Hsu (1982) and Gray and French (1990) have argued that this specification, which displays fat tails that shrink at an exponential rate and a high peak, provides a reasonably-good fit to stock return data. The density function of the exponential power distribution is given by

$$
f(x)=\frac{\exp \left[-\frac{1}{2}\left|\frac{x-\mu}{\alpha}\right|\left(\frac{2}{1+\beta}\right)\right]}{2^{\left(\frac{3+\beta}{2}\right)} \alpha \Gamma\left(\frac{3+\beta}{2}\right)},
$$


where $\mu(-\infty<\mu<\infty), \alpha(\alpha>0)$, and $\beta(-1<\beta \leq 1)$ are a location, a dispersion, and a shape parameter, respectively. This last parameter, in particular, measures the kurtosis of the distribution. More precisely, $\beta<0$ implies a platykurtic distribution, the Normal distribution is obtained when $\beta=0$, and fat tails and a high peak are obtained when $0<\beta \leq 1$, with the thickness of the tails increasing in $\beta{ }^{8}$ If $R_{t}$ follows an exponential power distribution, then $E\left(R_{t}\right)=\mu$ and $\operatorname{Var}\left(R_{t}\right)=\sigma^{2}=2^{(1+\beta)} \cdot \frac{\Gamma[3(1+\beta) / 2]}{\Gamma[(1+\beta) / 2]} \alpha^{2}$.

Mixtures of Two Normal Distributions. An alternative to assuming that stock returns are generated from a single distribution is to assume that they are generated by a mixture of distributions. Press (1967) argued that stock returns may be generated by the interaction of a continuous diffusion (Brownian motion) process and a discontinuous jump (Poison) process, where the former captures continuous changes in stock prices and the second models large informational shocks. Kon (1984) also argues in favor of (and finds empirical support for) this specification. The density function of a mixture of two Normal distributions is given by

$$
\begin{aligned}
f(x) & =\frac{1}{\sqrt{2 \pi \sigma_{1}^{2}}} \cdot e^{-\left[\frac{\left(x-\mu_{1}\right)^{2}}{2 \sigma_{1}^{2}}\right]}, \text { with probability } \lambda \\
& =\frac{1}{\sqrt{2 \pi \sigma_{2}^{2}}} \cdot e^{-\left[\frac{\left(x-\mu_{2}\right)^{2}}{2 \sigma_{2}^{2}}\right]}, \text { with probability }(1-\lambda),
\end{aligned}
$$

where $\mu_{i}\left(-\infty<\mu_{i}<\infty\right)$ and $\sigma_{i}^{2}\left(\sigma_{i}^{2}>0\right)$ are location and dispersion parameters, respectively. This mixture implies that stock returns are drawn from a Normal distribution with mean $\mu_{1}$ and standard deviation $\sigma_{1}$ with probability $\lambda$, and from a Normal distribution with mean $\mu_{2}$ and standard deviation $\sigma_{2}$ with probability $(1-\lambda)$. If $R_{t}$ follows such mixture of distributions, then $E\left(R_{t}\right)=\mu=\lambda \mu_{1}+(1-\lambda) \mu_{2}$ and $\left.\operatorname{Var}\left(R_{t}\right)=\sigma^{2}=\lambda\left\{\left(\mu_{1}-\mu\right)^{2}+\sigma_{1}{ }^{2}\right\}+(1-\lambda)\left\{\left(\mu_{2}-\mu\right)\right]^{2}+{\sigma_{2}}^{2}\right\}$. Of all the specifications we consider, this mixture of two Normal distributions is the only one that allows for skewness in the data. ${ }^{9}$

\footnotetext{
${ }^{8}$ For $\beta=1$, the double-exponential distribution is obtained.

${ }^{9}$ The coefficient of skewness $\left(k_{3}\right)$ that follows from the mixture of two Normal distributions is given by $k_{3}=\frac{\lambda\left[\left(\mu_{1}-\mu\right)^{2}+3\left(\mu_{1}-\mu\right) \sigma_{1}^{2}\right]+(1-\lambda)\left[\left(\mu_{2}-\mu\right)^{2}+3\left(\mu_{2}-\mu\right) \sigma_{2}^{2}\right]}{\left\{\lambda\left[\left(\mu_{1}-\mu\right)^{2}+\sigma_{1}^{2}\right]+(1-\lambda)\left[\left(\mu_{2}-\mu\right)^{2}+\sigma_{2}^{2}\right]\right\}^{3 / 2}}$.
} 


\section{IV- ESTIMATION OF PARAMETERS AND GOODNESS-OF-FIT TESTS}

We report in Table 3 below the (maximum-likelihood) estimations that result from fitting the theoretical distributions described in the previous part to the series of daily stock returns of the fourteen markets under consideration.

TABLE 3: Parameter Estimates

\begin{tabular}{|c|c|c|c|c|c|c|c|c|}
\hline & & AUS & BEL & DEN & ENG & FIN & FRA & GER \\
\hline \multirow[t]{2}{*}{$\mathrm{N}:$} & $\mu:$ & -0.00636 & -0.00205 & -0.00301 & 0.01804 & 0.03770 & -0.00258 & 0.00455 \\
\hline & $\sigma$ & 1.18440 & 0.71846 & 0.82253 & 0.81787 & 1.24302 & 0.99880 & 1.04666 \\
\hline \multirow[t]{2}{*}{$\mathrm{L}:$} & $\mu:$ & -0.00224 & 0.01136 & -0.00153 & 0.00871 & 0.01425 & 0.00330 & 0.01087 \\
\hline & $\alpha:$ & 0.58850 & 0.34741 & 0.41885 & 0.44202 & 0.66172 & 0.53471 & 0.53856 \\
\hline \multirow[t]{3}{*}{ S-t: } & $\mu:$ & 0.00441 & 0.01780 & -0.00072 & 0.00801 & 0.00629 & 0.00460 & 0.01237 \\
\hline & $\sigma:$ & 1.31263 & 0.75490 & 0.86401 & 0.80960 & 1.28016 & 0.99498 & 1.03513 \\
\hline & $v:$ & 2.88560 & 2.97920 & 3.34660 & 6.42820 & 4.26650 & 5.50990 & 4.28490 \\
\hline \multirow[t]{3}{*}{ EP: } & $\mu:$ & 0.00000 & 0.00000 & -0.00000 & 0.00045 & -0.00000 & 0.00000 & 0.00001 \\
\hline & $\alpha:$ & 0.39868 & 0.23524 & 0.28459 & 0.50094 & 0.51754 & 0.50065 & 0.42224 \\
\hline & $\beta:$ & 1.00000 & 1.00000 & 0.99999 & 0.50265 & 0.86572 & 0.69250 & 0.87113 \\
\hline \multirow[t]{7}{*}{$\mathrm{MN}:$} & $\mu_{1}:$ & 0.01638 & -0.24906 & -0.01645 & 0.20329 & -0.05409 & -0.23954 & 0.01268 \\
\hline & $\sigma_{1}:$ & 0.73017 & 1.63780 & 1.45330 & 1.85350 & 0.82717 & 2.05880 & 0.84158 \\
\hline & $\mu_{2}:$ & -0.09754 & 0.02832 & 0.00058 & 0.00638 & 0.23524 & 0.01831 & -0.11628 \\
\hline & $\sigma_{2}$ & 2.20880 & 0.49141 & 0.54173 & 0.70185 & 1.82780 & 0.84055 & 2.61410 \\
\hline & $\underline{\lambda}:$ & 0.80036 & 0.10946 & 0.21064 & 0.05918 & 0.68276 & 0.08102 & 0.93694 \\
\hline & $k_{3}:$ & -0.14149 & -0.51387 & -0.00157 & 0.18099 & 0.16450 & -0.19913 & -0.12140 \\
\hline & & ITA & NET & NOR & SPA & SWE & SWI & WOR \\
\hline \multirow[t]{2}{*}{$\mathrm{N}:$} & $\mu:$ & -0.00167 & 0.02405 & 0.00689 & -0.00205 & 0.02819 & 0.03055 & -0.00233 \\
\hline & $\sigma:$ & 1.36806 & 0.72673 & 1.32966 & 1.12401 & 1.24940 & 0.93056 & 0.65305 \\
\hline \multirow[t]{2}{*}{ L: } & $\mu:$ & 0.00150 & 0.03809 & -0.00854 & 0.00216 & 0.01305 & 0.04793 & -0.00399 \\
\hline & $\alpha:$ & 0.73705 & 0.39449 & 0.67926 & 0.58409 & 0.64014 & 0.48036 & 0.33609 \\
\hline \multirow[t]{3}{*}{ S- $t:$} & $\mu:$ & 0.00195 & 0.04037 & -0.01467 & 0.00289 & 0.00929 & 0.05299 & -0.00481 \\
\hline & $\sigma:$ & 1.38416 & 0.73395 & 1.30499 & 1.12765 & 1.27511 & 0.92339 & 0.66291 \\
\hline & $v:$ & 5.19850 & 5.68060 & 4.25530 & 4.22110 & 3.69760 & 4.28370 & 3.83590 \\
\hline \multirow[t]{3}{*}{ EP: } & $\mu:$ & -0.00000 & 0.03355 & -0.00000 & -0.00001 & 0.00000 & 0.03790 & -0.00319 \\
\hline & $\alpha:$ & 0.66812 & 0.41144 & 0.52770 & 0.45866 & 0.43700 & 0.38771 & 0.27211 \\
\hline & $\beta:$ & 0.72176 & 0.58354 & 0.88132 & 0.86788 & 1.00000 & 0.84330 & 0.84280 \\
\hline \multirow[t]{6}{*}{ MN: } & $\mu_{1}:$ & 0.00460 & -0.05175 & 0.00135 & 0.00379 & -0.00012 & -0.28008 & 0.01230 \\
\hline & $\sigma_{1}:$ & 1.66170 & 0.94692 & 1.06820 & 0.89482 & 0.91374 & 2.02840 & 1.20920 \\
\hline & $\mu_{2}:$ & -0.01178 & 0.08743 & 0.10965 & -0.06216 & 0.22347 & 0.06269 & -0.00536 \\
\hline & $\sigma_{2}:$ & 0.66026 & 0.45959 & 3.66050 & 2.45450 & 2.55450 & 0.72034 & 0.46024 \\
\hline & $\underline{\lambda}:$ & 0.61738 & 0.45537 & 0.94890 & 0.91149 & 0.87338 & 0.09374 & 0.17163 \\
\hline & $k_{3}:$ & 0.01064 & -0.17260 & 0.03141 & -0.05833 & 0.10426 & -0.37755 & 0.01247 \\
\hline
\end{tabular}

$\mathrm{N}=$ Normal; $\mathrm{L}=$ Logistic; S- $t=$ Scaled- $t$; EP $=$ Exponential Power; $\mathrm{MN}=$ Mixture of two Normal distributions. The coefficient of skewness $\left(k_{3}\right)$ follows from the expression in footnote 9 .

At least two things are worth noting from Table 3. First, recall that the Normal distribution and the $t$-distribution tend to converge as the degrees of freedom of the latter increase. However, the table shows that the estimated degrees of freedom of the scaled- $t$ distributions are very small in all markets (between 2.5 and 6.5), thus indicating that these empirical distributions diverge significantly from the Normal, particularly in the tails. 
Second, recall that the parameter $\beta$ of the exponential power distribution is a measure of its kurtosis, that for $\beta=0$ the Normal distribution is obtained, and that $\beta$ is increasing in the thickness of the tails (with an upper bound at $\beta=1$ ). Table 3 shows that $\beta$ is larger than .5 in all markets and larger than .8 in ten markets. This provides additional evidence of departures from normality, and, in particular, of the thickness of the tails of the empirical distributions under consideration.

In order to compare the relative fit of the theoretical distributions considered, we performed goodness-of-fit tests. ${ }^{10}$ To that purpose, we divided the range of returns into 20 equal, non-overlapping intervals contained in the range $[-10 \%, 10 \%]$. The results of these tests are shown below in Table 4.

TABLE 4: Goodness-of-fit Tests

\begin{tabular}{lrrrrrrrrrr} 
& $\mathrm{N}$ & $p$-value & $\mathrm{L}$ & $p$-value & $\mathrm{S}$ - $t$ & $p$-value & $\mathrm{EP}$ & $p$-value & $\mathrm{MN} p$-value \\
\hline \hline AUS & $1.8 \mathrm{e} 06$ & 0.0000 & $1,339.9$ & 0.0000 & 78.6 & $3.0 \mathrm{e}-10$ & 194.7 & 0.0000 & 101.1 & $2.9 \mathrm{e}-15$ \\
BEL & $9.3 \mathrm{e} 13$ & 0.0000 & $11,562.8$ & 0.0000 & 63.4 & $1.4 \mathrm{e}-07$ & $3,027.8$ & 0.0000 & 532.1 & 0.0000 \\
DEN & $1.3 \mathrm{e} 06$ & 0.0000 & 233.2 & 0.0000 & 14.7 & 0.5467 & 29.4 & 0.0214 & 30.7 & 0.0061 \\
ENG & $5.5 \mathrm{e} 06$ & 0.0000 & 296.9 & 0.0000 & 53.3 & $6.7 \mathrm{e}-06$ & 659.3 & 0.0000 & 19.0 & 0.1650 \\
FIN & 577.2000 & 0.0000 & 43.6 & 0.0004 & 16.8 & 0.3987 & 19.7 & 0.2340 & 16.6 & 0.2781 \\
FRA & $2.5 \mathrm{e} 09$ & 0.0000 & $1,863.4$ & 0.0000 & 93.1 & $6.7 \mathrm{e}-13$ & 994.9 & 0.0000 & 106.8 & $2.2 \mathrm{e}-16$ \\
GER & $34,344.3000$ & 0.0000 & 397.2 & 0.0000 & 56.1 & $2.3 \mathrm{e}-06$ & 103.1 & $9.1 \mathrm{e}-15$ & 45.6 & $3.2 \mathrm{e}-05$ \\
ITA & $1.2 \mathrm{e} 06$ & 0.0000 & 322.1 & 0.0000 & 114.7 & $1.1 \mathrm{e}-16$ & 181.5 & 0.0000 & $7,214.9$ & 0.0000 \\
NET & 939.6000 & 0.0000 & 30.0 & 0.0263 & 12.3 & 0.7231 & 20.5 & 0.1985 & 30.9 & 0.0057 \\
NOR & $2.2 \mathrm{e} 10$ & 0.0000 & 749.5 & 0.0000 & 21.5 & 0.1601 & 111.1 & $2.2 \mathrm{e}-16$ & 19.1 & 0.1612 \\
SPA & $5.5 \mathrm{e} 09$ & 0.0000 & $3,595.4$ & 0.0000 & 55.9 & $2.5 \mathrm{e}-06$ & 486.7 & 0.0000 & 100.7 & $3.4 \mathrm{e}-15$ \\
SWE & $2.2 \mathrm{e} 09$ & 0.0000 & $1,351.9$ & 0.0000 & 20.7 & 0.1903 & 94.8 & $3.2 \mathrm{e}-13$ & 40.7 & 0.0002 \\
SWI & $5.9 \mathrm{e} 11$ & 0.0000 & $33,157.4$ & 0.0000 & 185.6 & 0.0000 & $4,179.7$ & 0.0000 & 356.9 & 0.0000 \\
WOR & $1.7 \mathrm{e} 06$ & 0.0000 & 189.2 & 0.0000 & 6.4 & 0.9832 & 40.5 & 0.0007 & 18.2 & 0.1978 \\
\hline \hline
\end{tabular}

$\mathrm{N}=$ Normal; $\mathrm{L}=$ Logistic; $\mathrm{S}-t=$ Scaled- $;$ EP = Exponential Power; $\mathrm{MN}=$ Mixture of two Normal distributions. The goodness of fit test follows a Chi-square distribution with $p-k-1$ degrees of freedom, where $p$ is the number of intervals and $k$ is the number of parameters estimated for each distribution. Degrees of freedom are 17 for $N$ and $\mathrm{L}, 16$ for $\mathrm{S}-t$ and $\mathrm{EP}$, and 14 for $\mathrm{MN}$.

This table shows that, as expected, the Normal distribution provides the worst fit among all the specifications considered; it is clearly rejected in all markets. The logistic distribution does not fit much better than the Normal, and is also rejected (at the 5\% significance level) in all markets. The exponential power distribution also provides a very poor fit, being rejected (at the 5\% significance level) in twelve of the fourteen markets analyzed.

\footnotetext{
${ }^{10}$ We do not use the likelihood-ratio test for the obvious reason that not all these theoretical distributions are nested within each other; hence, their log-likelihood functions are not comparable.
} 
Table 4 shows partial support for a mixture of two Normal distributions. This specification cannot be rejected (at the $5 \%$ significance level) in four markets, and is the one that best fits three markets. ${ }^{11}$ Finally, the scaled- $t$ is the distribution that provides the best overall fit among all the specifications considered. This distribution cannot be rejected (at the $5 \%$ significance level) in six markets, and is the one that best fits ten of the fourteen markets analyzed. ${ }^{12}$

It may be interesting to contrast our results with those reported by Peiró (1994), who, although using different indices and sample periods, also studies the English, German, French, and Spanish markets. In general, his results seem to be more optimistic than ours. He finds, for example, that the scaled- $t$ distribution cannot be rejected in any of these four markets; we reject it in all four. He also finds that neither the logistic, nor the scaled-t, nor the mixture of two Normal distributions can be rejected in the German market; we reject all three specifications. And he finds that the French market is best fitted by an exponential power distribution, although we clearly reject such specification.

The interesting issue is whether these different results stem from distributions that change significantly over time. Peiró (1994) uses two years of data we do not use (1988-9), we use two years of data he does not use (1993-4), and we overlap in three years (1990-2). Thus, if the differences between our results and his stem from changes in the distributions of stock returns, we should then be wary of using statistical models that assume that such distributions are time invariant. It may be the case that, contrary to such assumption, stock returns distributions may actually be changing rapidly over time.

\section{V- ERRORS IMPLIED BY THE NORMALITY ASSUMPTION}

The tests of normality reported in part II establish that the distributions of stock returns of the fourteen markets analyzed exhibit significant departures from normality. In addition, the goodness-of-fit tests reported in part IV establish that a scaled- $t$ distribution and a mixture of two Normal distributions provide a significantly-better fit than the Normal

\footnotetext{
"Kon (1984) fits mixtures of up to five Normal distributions to the thirty stock of the Dow Jones Industrial Average. He finds that a mixture of four Normal distributions best fits seven stocks, a mixture of thrce Normal distributions best fits eleven stocks, and a mixture of two Normal distributions best fits the remaining twelve stocks.

${ }^{12}$ All five specifications considered are clearly rejected in the Swiss market. The fact that (as Table 1 shows) the Swiss market is the one that exhibits the largest degree of skewness may perhaps explain this finding.
} 
distribution. In this part, we quantify the error that can be made by predicting the probability of obtaining returns in specified intervals by assuming an underlying Normal distribution.

In order to assess this error, we first estimate the (unconditional) probability of obtaining returns in a given interval using the parameters previously estimated (and reported in Table 3) for the Normal distribution; we subsequently repeat this process for the twelve intervals we consider. Then we estimate the same probability using the parameters previously estimated (and reported in Table 3) for the scaled-t distribution for the same twelve intervals. ${ }^{13}$ We finally compare, one by one, the probability of obtaining returns in each interval. The results of our estimations are reported below in Table 5.

TABLE 5: Probabilities of Obtaining Returns in Specified Intervals

\begin{tabular}{|c|c|c|c|c|c|c|c|}
\hline \multirow{3}{*}{ AUS: } & \multirow{3}{*}{$\begin{array}{l}\mathrm{N}: \\
\mathrm{S}-t:\end{array}$} & \multirow{2}{*}{$\frac{[\bar{x}, \bar{x}+s]}{0.34144}$} & \multicolumn{2}{|c|}{$[\bar{x}+s, \bar{x}+2 s][\bar{x}+2 s, \bar{x}+3 s]$} & \multicolumn{3}{|c|}{$[\bar{x}+3 s, \bar{x}+4 s][\bar{x}+4 s, \bar{x}+5 s][\bar{x}+5 s, \bar{x}+6 s]$} \\
\hline & & & 0.13585 & 0.02136 & 0.00131 & 0.00003 & $2.83 \mathrm{e}-7$ \\
\hline & & 0.40124 & 0.07899 & 0.01617 & 0.00488 & 0.00192 & 0.00090 \\
\hline \multirow[t]{2}{*}{ BEL: } & $\mathrm{N}:$ & 0.34144 & 0.13585 & 0.02136 & 0.00131 & 0.00003 & $2.83 \mathrm{e}-7$ \\
\hline & S- $t$ : & 0.41415 & 0.07917 & 0.01542 & 0.00448 & 0.00171 & 0.00079 \\
\hline \multirow[t]{2}{*}{ DEN: } & $\mathrm{N}:$ & 0.34144 & 0.13585 & 0.02136 & 0.00131 & 0.00003 & $2.83 \mathrm{e}-7$ \\
\hline & S-t: & 0.39052 & 0.08591 & 0.01703 & 0.00472 & 0.00170 & 0.00074 \\
\hline \multirow[t]{2}{*}{ ENG: } & $N:$ & 0.34144 & 0.13585 & 0.02136 & 0.00131 & 0.00003 & $2.83 \mathrm{e}-7$ \\
\hline & $\mathrm{S}-t:$ & 0.34208 & 0.11980 & 0.02565 & 0.00539 & 0.00131 & 0.00038 \\
\hline \multirow[t]{2}{*}{ FIN: } & $\mathrm{N}:$ & 0.34144 & 0.13585 & 0.02136 & 0.00131 & 0.00003 & $2.83 \mathrm{e}-7$ \\
\hline & S-t: & 0.36763 & 0.09442 & 0.01837 & 0.00448 & 0.00140 & 0.00053 \\
\hline \multirow[t]{2}{*}{ FRA: } & $N:$ & 0.34144 & 0.13585 & 0.02136 & 0.00131 & 0.00003 & $2.83 \mathrm{e}-7$ \\
\hline & S- $t:$ & 0.37242 & 0.10624 & 0.01932 & 0.00394 & 0.00101 & 0.00031 \\
\hline \multirow[t]{2}{*}{ GER: } & $\mathrm{N}:$ & 0.34144 & 0.13585 & 0.02136 & 0.00131 & 0.00003 & $2.83 e-7$ \\
\hline & S-t: & 0.38555 & 0.09478 & 0.01736 & 0.00407 & 0.00124 & 0.00046 \\
\hline \multirow[t]{2}{*}{ ITA: } & $\mathrm{N}:$ & 0.34144 & 0.13585 & 0.02136 & 0.00131 & 0.00003 & $2.83 \mathrm{e}-7$ \\
\hline & S-t: & 0.37016 & 0.10536 & 0.01974 & 0.00423 & 0.00114 & 0.00037 \\
\hline \multirow[t]{2}{*}{ NET: } & $\mathrm{N}:$ & 0.34144 & 0.13585 & 0.02136 & 0.00131 & 0.00003 & $2.83 e-7$ \\
\hline & S- $t:$ & 0.37219 & 0.11176 & 0.02080 & 0.00421 & 0.00106 & 0.00032 \\
\hline \multirow[t]{2}{*}{ NOR } & $\mathrm{N}:$ & 0.34144 & 0.13585 & 0.02136 & 0.00131 & 0.00003 & $2.83 e-7$ \\
\hline & S- $t:$ & 0.37961 & 0.08966 & 0.01630 & 0.00385 & 0.00118 & 0.00044 \\
\hline \multirow[t]{2}{*}{ SPA: } & $\mathrm{N}:$ & 0.34144 & 0.13585 & 0.02136 & 0.00131 & 0.00003 & $2.83 e-7$ \\
\hline & S- $t:$ & 0.38255 & 0.09533 & 0.01784 & 0.00427 & 0.00132 & 0.00050 \\
\hline \multirow[t]{2}{*}{ SWE: } & $\mathrm{N}:$ & 0.34144 & 0.13580 & 0.02136 & 0.00131 & 0.00003 & $2.83 e-7$ \\
\hline & S-t: & 0.38109 & 0.08683 & 0.01676 & 0.00438 & 0.00149 & 0.00061 \\
\hline \multirow[t]{2}{*}{ SWI: } & $\mathrm{N}:$ & 0.34144 & 0.13585 & 0.02136 & 0.00131 & 0.00003 & $2.83 \mathrm{e}-7$ \\
\hline & S- $t:$ & 0.39018 & 0.09796 & 0.01799 & 0.00421 & 0.00128 & 0.00048 \\
\hline \multirow[t]{2}{*}{ WOR: } & N: & 0.34144 & 0.13585 & 0.02136 & 0.00131 & 0.00003 & $2.83 \mathrm{e}-7$ \\
\hline & S-t: & 0.38354 & 0.09017 & 0.01723 & 0.00438 & 0.00145 & 0.00058 \\
\hline
\end{tabular}

$\mathrm{N}=$ Normal; S- $t=$ Scaled- $t$. Each number shows the probability of obtaining a return in the specified interval under the specified distribution. Each distribution is centered around its sample mean $(\bar{x})$, and the length of each interval is equal to each distribution's sample standard deviation $(s)$, both taken from Table 1. Both $N$ and S- $t$ are symmetric distributions; hence, predictions are reported only for one half of each distribution.

\footnotetext{
${ }^{13}$ We report results for the scaled- $t$ distribution for all markets because the difference between the probability predicted by such specification, and that predicted by the mixture of two Normals in the markets for which the latter provides a better fit (ENG, GER, NOR) is negligible.
} 
Table 5 shows that the probability of obtaining returns in any given interval is very different depending on whether the Normal or the scaled-t are assumed as the underlying distribution. Recall that leptokurtic distributions have a high peak, thus exhibiting clustering of observations around the mean. Accordingly, Table 5 shows that the probability of obtaining returns one standard deviation around the mean is higher under the scaled-t distribution than under the Normal distribution in all markets. Furthermore, note that the opposite is the case in the intervals $[\bar{x}+s, \bar{x}+2 s]$ and $[\bar{x}+2 s, \bar{x}+3 s]$; that is, the probability of obtaining returns in both intervals is higher under the Normal distribution than under the scaled- $t$ distribution in all markets.

Note, however, that the situation reverses again for the interval $[\bar{x}+3 s, \bar{x}+4 s]$ and all intervals beyond. In other words, the probability of obtaining returns in any of these intervals is higher under the scaled-t distribution than under the Normal distribution in all markets. Furthermore, note that the difference between the probability predicted by each distribution increases dramatically as we move away from the mean. To illustrate, the probability of obtaining a return between three and four standard deviations away from the mean is, on average, over three times higher (3.4) under the scaled-t distribution; the probability of obtaining a return between four and five standard deviations is, on average, over forty five times higher (45.7) under the scaled-t distribution; and the probability of obtaining a return between five and six standard deviations is, on average, almost two thousand times higher $(1,870)$ under the scaled- $t$ distribution.

The previous results show that investors that predict the probability of obtaining returns in specified intervals by assuming an underlying Normal distribution may significantly underestimate the risk of investing in European stocks. This underestimation, as the numbers above show, is particularly severe in the tails of the distribution; that is, when predicting the probability of large (positive or negative) returns. Returns three and more standard deviations away from the mean, which occur with a negligible probability under the Normal distribution, occur much more frequently under a scaled-t distribution.

Another way to look at this issue is by comparing the number of outliers that would be expected under a Normal distribution with those expected under a scaled- $t$ distribution. Table 6 below reports these numbers, as well as the observed number of outliers in each of the markets considered. For the purposes of the table, we consider outliers those returns at least three standard deviations away from the mean. 
TABLE 6: Expected and Observed Outliers

\begin{tabular}{|c|c|c|c|c|c|c|c|c|c|c|c|c|}
\hline \multirow{2}{*}{ Market } & \multicolumn{3}{|c|}{$>3 s$} & \multicolumn{3}{|c|}{$>4 s$} & \multicolumn{3}{|c|}{$>5 s$} & \multicolumn{3}{|c|}{$>6 s$} \\
\hline & $\mathrm{N}$ & S-t & Obs & $\mathrm{N}$ & S-t & Obs & $\mathrm{N}$ & $\mathrm{S}-t$ & Obs & $\mathrm{N}$ & $\mathrm{S}-t$ & Obs \\
\hline AUS & 1.75 & 11.78 & 11 & 0.04 & 5.42 & 7 & 0.0004 & 2.92 & 3 & 0.0000 & 1.75 & 0 \\
\hline BEL & 1.75 & 10.57 & 6 & 0.04 & 4.72 & 3 & 0.0004 & 2.49 & 2 & 0.0000 & 1.46 & 2 \\
\hline DEN & 1.75 & 10.54 & 9 & 0.04 & 4.39 & 4 & 0.0004 & 2.17 & 2 & 0.0000 & 1.21 & 0 \\
\hline ENG & 1.75 & 9.52 & 8 & 0.04 & 2.49 & 3 & 0.0004 & 0.77 & 2 & 0.0000 & 0.28 & 1 \\
\hline FIN & 1.75 & 9.00 & 11 & 0.04 & 3.16 & 3 & 0.0004 & 1.33 & 0 & 0.0000 & 0.65 & 0 \\
\hline FRA & 1.75 & 7.13 & 7 & 0.04 & 1.99 & 1 & 0.0004 & 0.68 & 1 & 0.0000 & 0.27 & 0 \\
\hline GER & 1.75 & 8.09 & 8 & 0.04 & 2.77 & 3 & 0.0004 & 1.16 & 3 & 0.0000 & 0.55 & 0 \\
\hline ITA & 1.75 & 7.83 & 7 & 0.04 & 2.32 & 0 & 0.0004 & 0.84 & 0 & 0.0000 & 0.35 & 0 \\
\hline NET & 1.75 & 7.56 & 3 & 0.04 & 2.07 & 1 & 0.0004 & 0.69 & 0 & 0.0000 & 0.27 & 0 \\
\hline NOR & 1.75 & 7.68 & 5 & 0.04 & 2.66 & 4 & 0.0004 & 1.12 & 2 & 0.0000 & 0.54 & 2 \\
\hline SPA & 1.75 & 8.54 & 7 & 0.04 & 2.98 & 4 & 0.0004 & 1.26 & 2 & 0.0000 & 0.61 & 1 \\
\hline SWE & 1.75 & 9.33 & 12 & 0.04 & 3.62 & 5 & 0.0004 & 1.68 & 3 & 0.0000 & 0.89 & 1 \\
\hline SWI & 1.75 & 8.35 & 7 & 0.04 & 2.86 & 2 & 0.0004 & 1.19 & 1 & 0.0000 & 0.57 & 0 \\
\hline WOR & 1.75 & 9.17 & 8 & 0.04 & 3.46 & 4 & 0.0004 & 1.56 & 2 & 0.0000 & 0.80 & 1 \\
\hline
\end{tabular}

$\mathrm{N}=$ Normal; S- $t=$ Scaled $t$; Obs $=$ Observed; $s=$ sample standard deviation. Numbers under $\mathrm{N}$ and S- $t$ show the expected number of outliers under each Normal and Scaled- $t$ distribution, respectively. Numbers under Obs show the observed number of outliers under each empirical distribution.

Table 6 shows that, as expected, the Normal distribution consistently underestimates the expected number of outliers in all markets. The scaled- $t$ distribution, on the other hand, provides a much better (though not perfect) prediction of the expected number of outliers. Furthermore, this distribution (unlike the Normal) does not display a systematic bias in its predictions; that is, the predicted number of outliers is in some cases higher and in some cases lower than the observed number of outliers. These results, as well as those discussed above, show that the normality assumption unequivocally leads investors to underestimate the risk of investing in European stocks.

As stated above, the argument for normally-distributed stock returns stems from the random walk theory of stock prices. Using the same data of this study, and consistent with the results just discussed, Estrada (1997) finds that investors who mistakenly assume that stock prices follow a random walk (and use the implications of such theory) may underestimate the risk of investing in European stocks by an average of $1.25 \%$ a month. Certainly, an argument can be made against using simplifying assumptions that may lead investors to make such substantial mistakes.

\section{VI- THE DISTRIBUTION OF MONTHLY STOCK RETURNS}

We examine in this part whether the departures from normality observed in daily stock returns are also observed in monthly stock returns. To that purpose, we compute monthly stock returns $\left(M R_{t}\right)$ as $M R_{t}=100\left[\ln \left(I_{k}\right)-\ln \left(I_{k-1}\right)\right]$, where $I_{k}$ and $I_{k-1}$ are the value of the 
index on the last day of month $k$ and the last day of month $k-1$, respectively. Table 7 below summarizes some relevant information about the empirical distributions of monthly stock returns of the fourteen markets under consideration.

TABLE 7: Sample Moments of the Distributions of Monthly Stock Returns

\begin{tabular}{lrrrrrrrr}
\hline \hline Market & Mean & \multicolumn{1}{c}{ SD } & \multicolumn{1}{c}{ Min } & \multicolumn{1}{c}{ Max } & \multicolumn{1}{c}{ Skw } & \multicolumn{1}{c}{ SSkw } & \multicolumn{1}{c}{ Krt } & \multicolumn{1}{c}{ SKrt } \\
\hline AUS & -0.1384 & 8.3512 & -25.4979 & 20.2526 & -0.3408 & -1.0776 & 1.5646 & 2.4739 \\
BEL & -0.0445 & 4.6504 & -12.2494 & 13.2179 & -0.2539 & -0.8028 & 1.0643 & 1.6828 \\
DEN & -0.0655 & 5.1179 & -11.0931 & 11.6782 & -0.0524 & -0.1658 & -0.3183 & -0.5032 \\
ENG & 0.3923 & 4.7308 & -8.6594 & 10.4902 & 0.0773 & 0.2445 & -0.3561 & -0.5631 \\
FIN & 0.8200 & 8.7771 & -20.4114 & 20.9633 & 0.0505 & 0.1596 & -0.1234 & -0.1951 \\
FRA & -0.0560 & 5.4079 & -14.7757 & 11.9082 & -0.2230 & -0.7051 & 0.0088 & 0.0139 \\
GER & 0.0993 & 5.6230 & -19.7969 & 9.3121 & -1.0676 & -3.3760 & 2.5415 & 4.0185 \\
ITA & -0.0363 & 7.4437 & -16.1316 & 20.8624 & 0.3993 & 1.2626 & 0.1139 & 0.1801 \\
NET & 0.5231 & 3.9566 & -9.9611 & 9.0035 & -0.0950 & -0.3006 & -0.3188 & -0.5041 \\
NOR & 0.1498 & 6.8552 & -16.4028 & 12.6052 & -0.3229 & -1.0210 & -0.4515 & -0.7139 \\
SPA & -0.0446 & 6.4968 & -20.7705 & 12.8100 & -0.4144 & -1.3104 & 0.4918 & 0.7775 \\
SWE & 0.6132 & 7.6259 & -23.9370 & 23.0205 & -0.2962 & -0.9366 & 1.5848 & 2.5057 \\
SWI & 0.6646 & 4.8953 & -14.5733 & 12.0524 & -0.5547 & -1.7542 & 1.6346 & 2.5845 \\
WOR & -0.0507 & 4.0607 & -12.9982 & 9.7972 & -0.5708 & -1.8051 & 1.5585 & 2.4642 \\
\hline \hline
\end{tabular}

Sample size $=60$ for all markets. Mean returns, standard deviations (SD), minimum returns (Min), and maximum returns (Max) are all expressed in percentages. Skw $=$ Skewness $=m_{3} / s^{3}$ and Krt $=$ Kurtosis $=m_{4} / s^{4}-3$, where $m_{i}$ and $s$ are the $i$ th central sample moment and the sample standard deviation of each distribution, respectively; both coefficients are computed with a finite-sample adjustment. SSkw = Standardized skewness and SKrt $=$ Standardized kurtosis.

The columns labeled SSkw and SKrt in Table 7 show the standardized coefficients of skewness and kurtosis for monthly stock returns. At the 5\% significance level, these coefficients show, unlike those from Table 1, that only one distribution displays a significant degree of skewness (GER), and five distributions (AUS, GER, SWE, SWI, WOR) display a significant degree of kurtosis. In other words, the departures from normality measured by the standardized coefficients of skewness and kurtosis are far less evident in monthly stock returns than in daily stock returns. ${ }^{14}$

In order to evaluate more formally the plausibility of the normality assumption as it applies to monthly stock returns, we rerun the three tests of normality run above in part II for daily stock returns. The results of these tests for monthly data are reported below in Table 8.

\footnotetext{
${ }^{14}$ Campbell, Lo, and MacKinlay (1997) compare the daily and monthly distributions of stock returns for two indices and ten stocks from the U.S. for the period 1962-94. Predictably, they find that the skewness and kurtosis displayed by the monthly distributions is significantly lower than those displayed by the daily distributions.
} 
TABLE 8: Tests of Normality (Monthly Stock Returns)

\begin{tabular}{|c|c|c|c|c|c|c|c|}
\hline \multirow[b]{2}{*}{ Market } & \multicolumn{3}{|c|}{ Goodness of Fit } & \multicolumn{2}{|c|}{ Kolmogorov-Smirnov } & \multicolumn{2}{|c|}{ Jarque-Bera } \\
\hline & Statistic & $\mathrm{df}$ & $p$-value & Statistic & $p$-value & Statistic & $p$-value \\
\hline AUS & 6.0487 & 4 & 0.1955 & 0.0958 & 0.6410 & 7.2814 & 0.0262 \\
\hline BEL & 5.3849 & 5 & 0.3707 & 0.1169 & 0.3851 & 3.4765 & 0.1758 \\
\hline DEN & 3.9205 & 5 & 0.5609 & 0.0622 & 0.9745 & 0.2807 & 0.8690 \\
\hline ENG & 7.3708 & 6 & 0.2879 & 0.0715 & 0.9190 & 0.3768 & 0.8283 \\
\hline FIN & 5.1417 & 4 & 0.2731 & 0.0873 & 0.7498 & 0.0636 & 0.9687 \\
\hline FRA & 3.0409 & 4 & 0.5510 & 0.0755 & 0.8835 & 0.4975 & 0.7798 \\
\hline GER & 3.8972 & 5 & 0.5643 & 0.0910 & 0.7035 & 27.5458 & 0.0000 \\
\hline ITA & 5.2280 & 4 & 0.2647 & 0.0804 & 0.8324 & 1.6268 & 0.4433 \\
\hline NET & 4.4380 & 5 & 0.4882 & 0.0647 & 0.9630 & 0.3443 & 0.8418 \\
\hline NOR & 4.8551 & 5 & 0.4338 & 0.0977 & 0.6153 & 1.5523 & 0.4602 \\
\hline SPA & 2.6113 & 5 & 0.7596 & 0.0652 & 0.9608 & 2.3219 & 0.3132 \\
\hline SWE & 2.4648 & 4 & 0.6509 & 0.0630 & 0.9712 & 7.1563 & 0.0279 \\
\hline SWI & 3.8341 & 5 & 0.5735 & 0.1025 & 0.5534 & 9.7567 & 0.0076 \\
\hline WOR & 5.0296 & 5 & 0.4123 & 0.1043 & 0.5308 & 9.3304 & 0.0094 \\
\hline
\end{tabular}

The goodness-of-fit test follows a Chi-square distribution with the degrees of freedom (df) indicated above. The asymptotic critical value for the Kolmogorov-Smirnov test at the $5 \%$ significance level is 0.176 . The Jarque-Bera test is asymptotically distributed as a Chi-square with 2 degrees of freedom; its critical value at the $5 \%$ significance level is 5.99 .

Table 8 shows that normality does seem to be a reasonable assumption for monthly stock returns. At the 5\% significance level, the null hypothesis of normality is rejected in no market under the goodness-of-fit test and Kolmogorov-Smirnov test, and in five markets under the Jarque-Bera test. These results, though significantly different from those reported in Table 2 for daily data, should not be entirely surprising. Under the central limit theorem, the longer the time interval for which returns are computed, the more the resulting distribution should conform to the Normal distribution.

\section{VII- CONCLUSIONS}

The evidence against the assumption that daily stock returns are normally distributed has been mounting for over thirty years. Most of the empirical evidence analyzes U.S. data, although some recent studies have considered European markets. In this article, we used data from the first half of the decade to test the hypothesis that stock returns in thirteen European markets are normally distributed.

We started by describing the data and testing the hypothesis of normality. Not surprisingly, the distributions of daily stock returns analyzed show fat tails and high peaks, as well as skewness in different directions. These results are fully consistent with those found for many other markets and reported in many other studies.

We then fitted the Normal distribution to the data, as well as four alternative specifications, all of which exhibit fat tails and one that also allows for skewness. 
Predictably, we found that the Normal distribution exhibited the worst fit in all markets. We also found that neither the logistic nor the exponential power distributions provide a good fit to the empirical distributions of European stock returns. However, we found partial support for a mixture of two normal distributions, which cannot be rejected in four markets and best fits three markets, and overall support for the scaled- $t$ distribution, which cannot be rejected in six markets and best fits ten of the fourteen markets analyzed.

We also attempted to quantify the error that can be made by predicting the probability of obtaining returns in specified intervals by using the Normal distribution instead of the more appropriate specification. We have shown that such errors can be very large, particularly in the tails, and that the Normal distribution (unlike the scaled- $t$ distribution) consistently underestimates the probability of (positive or negative) large returns. Therefore, we can argue that booms and crashes in European markets are much more likely to occur than what a Normal distribution would predict.

Finally, we examined whether the departures from normality observed for daily data are also observed in monthly data. As expected under the central limit theorem, we found that this is not the case. The data showed that the Normal distribution may in fact be a reasonable approximation to the empirical distributions of European monthly stock returns. 


\section{APPENDIX}

A1- MARKET BEHAVIOR
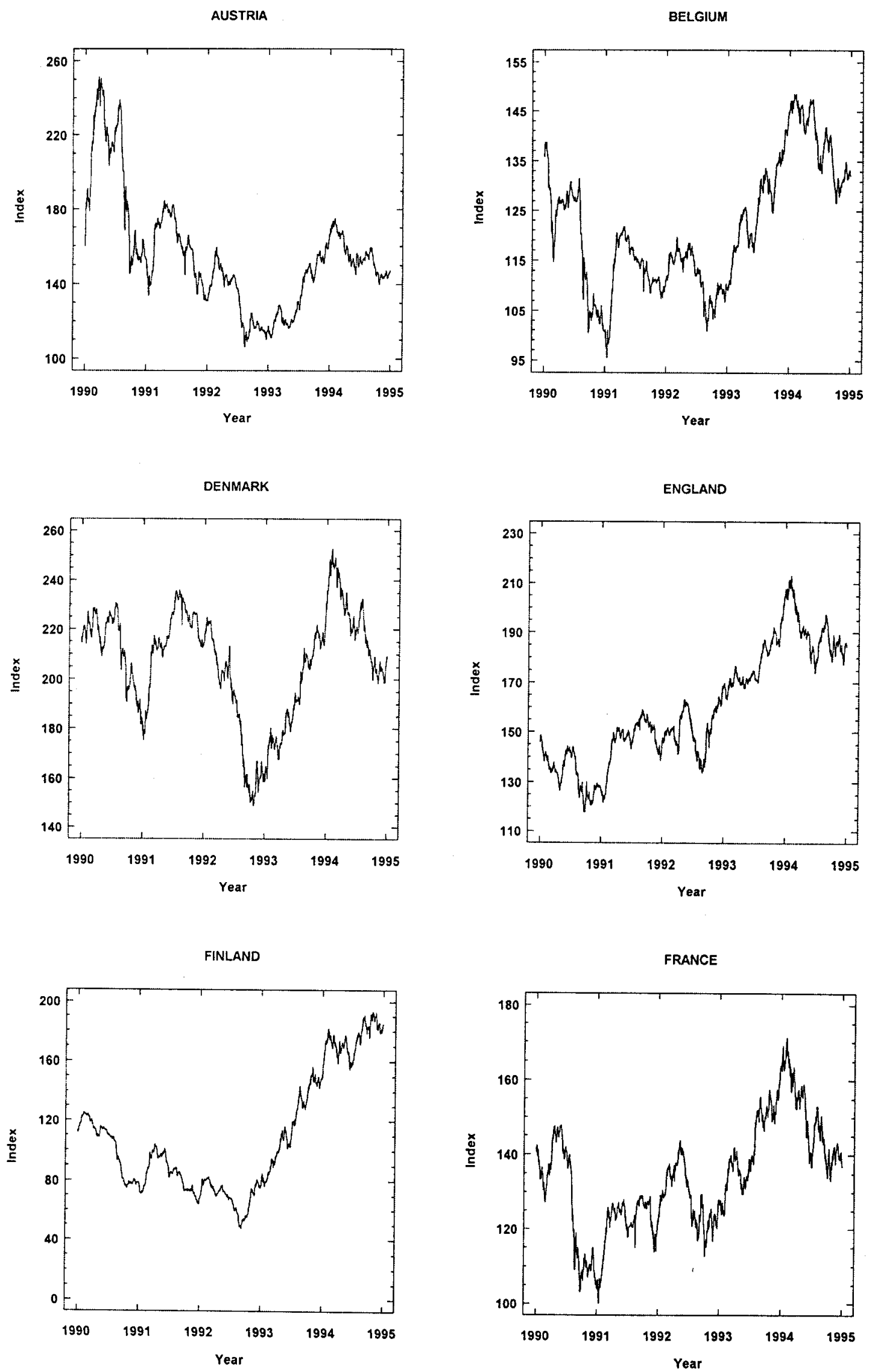

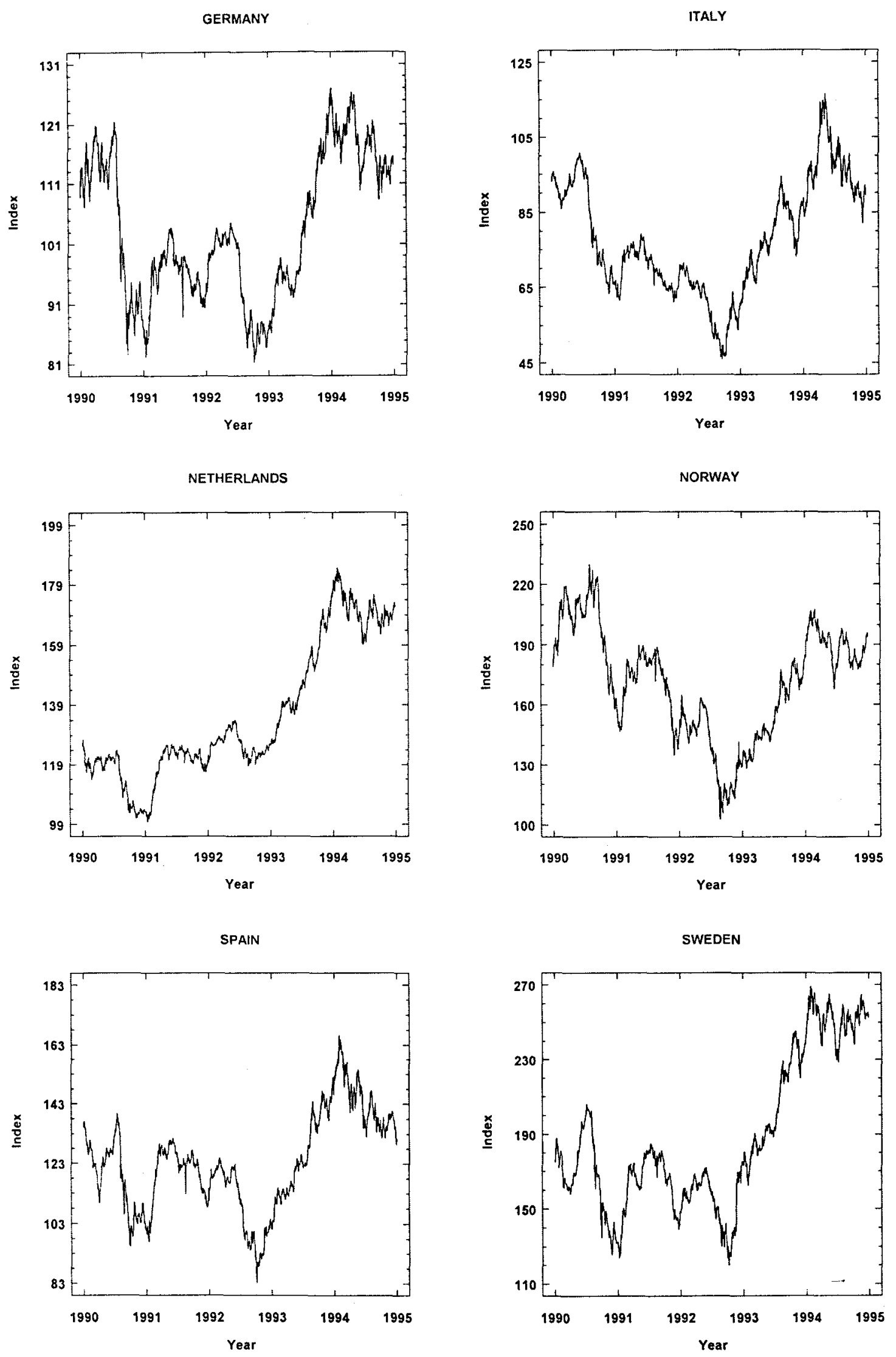

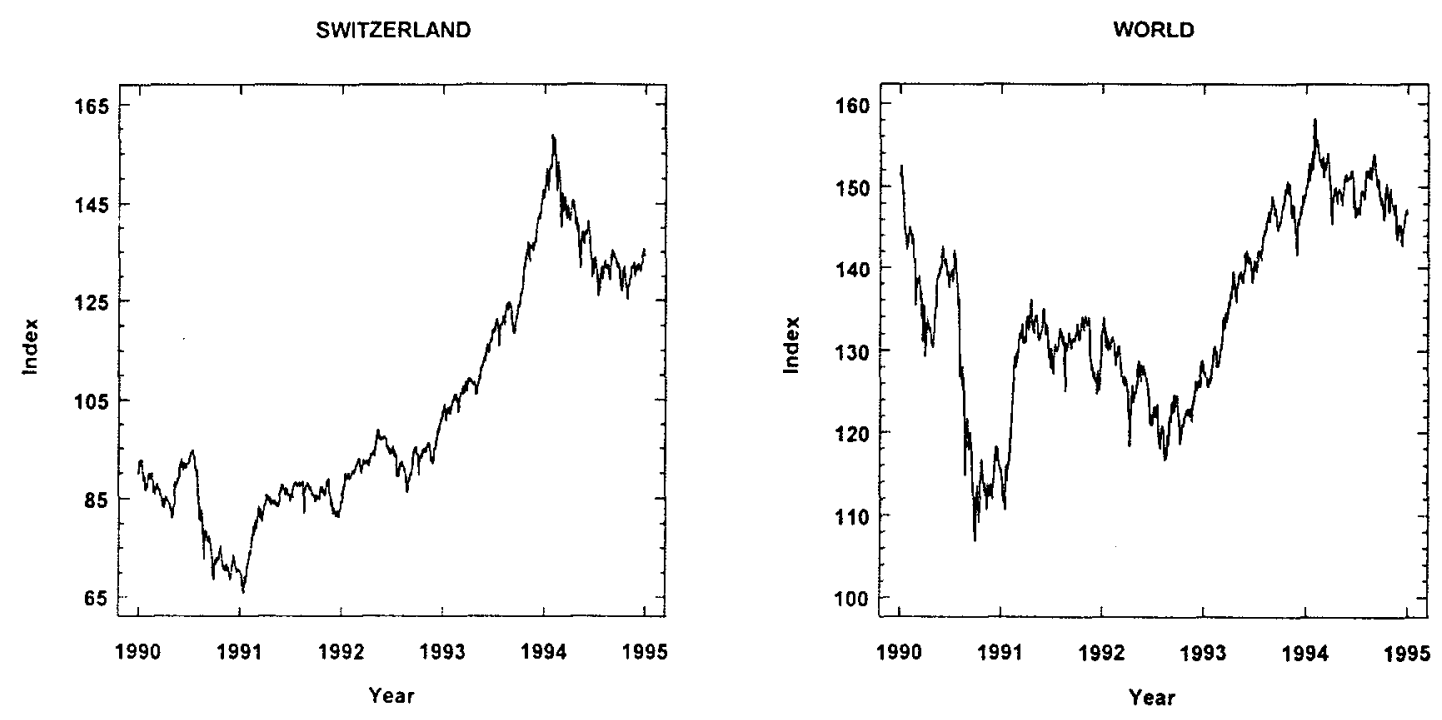

\section{A2- HISTOGRAMS}
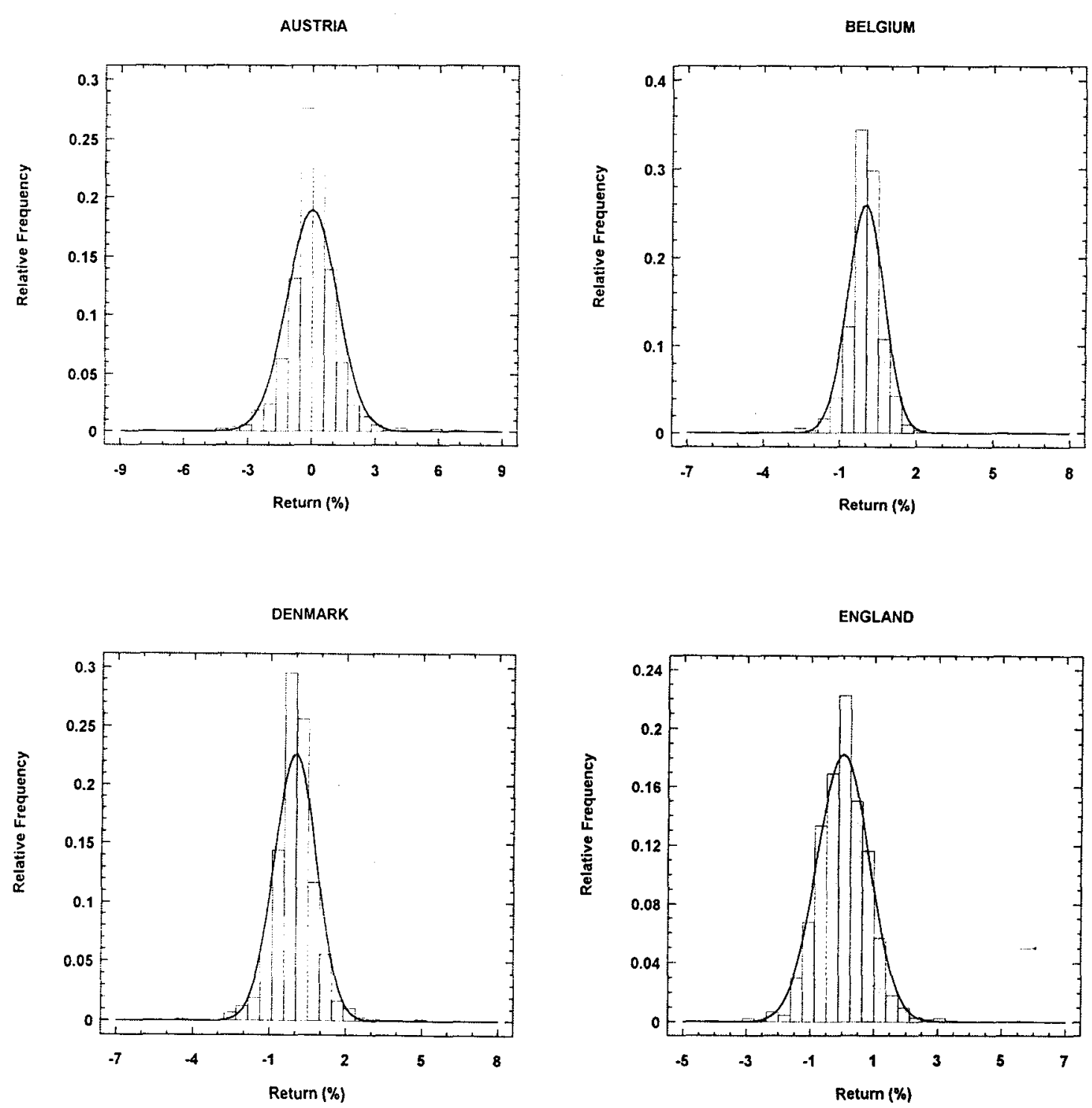
FINLAND

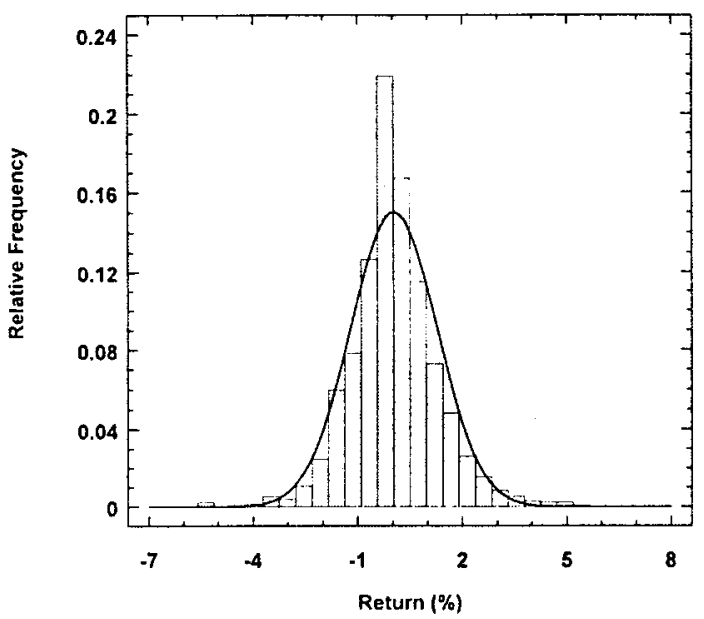

GERMANY

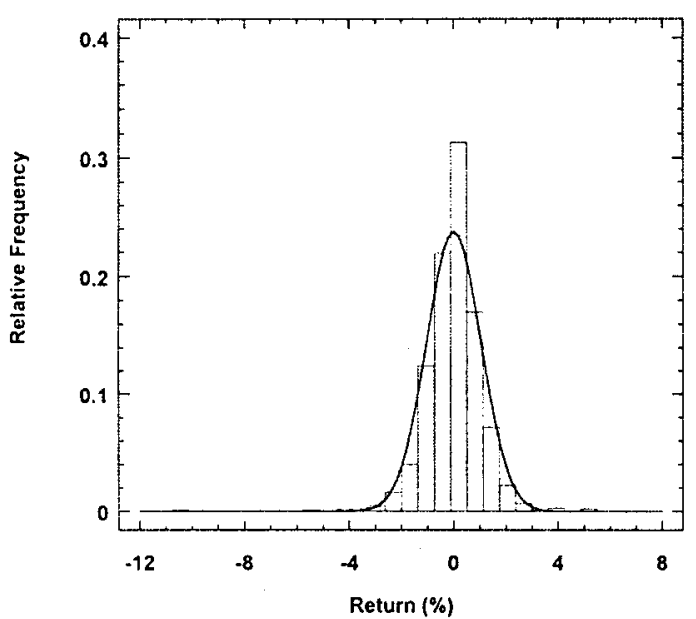

NETHERLANDS

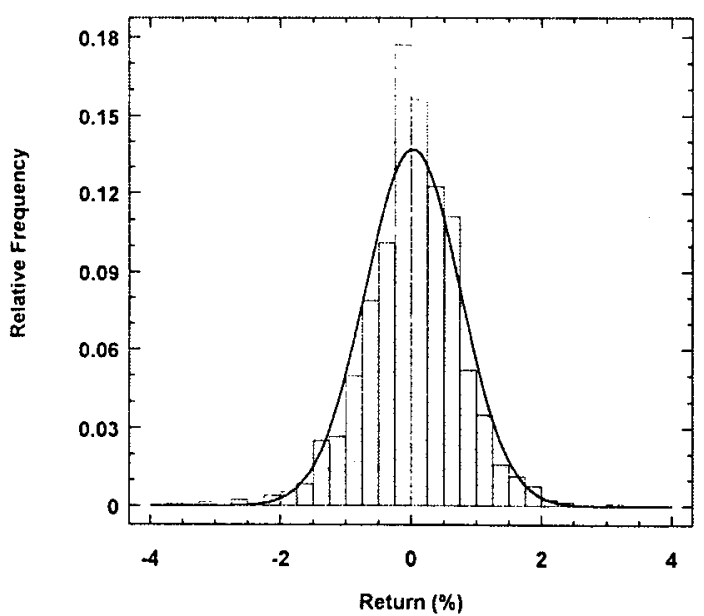

FRANCE

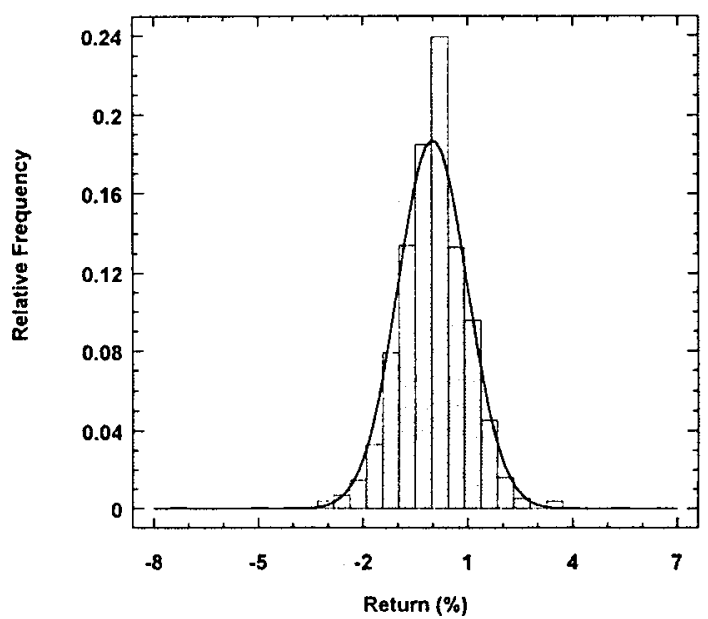

ITALY

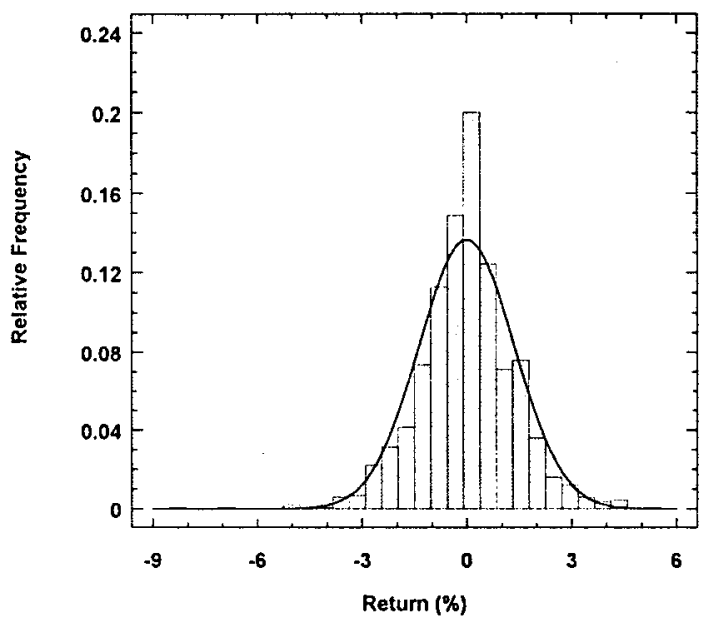

NORWAY

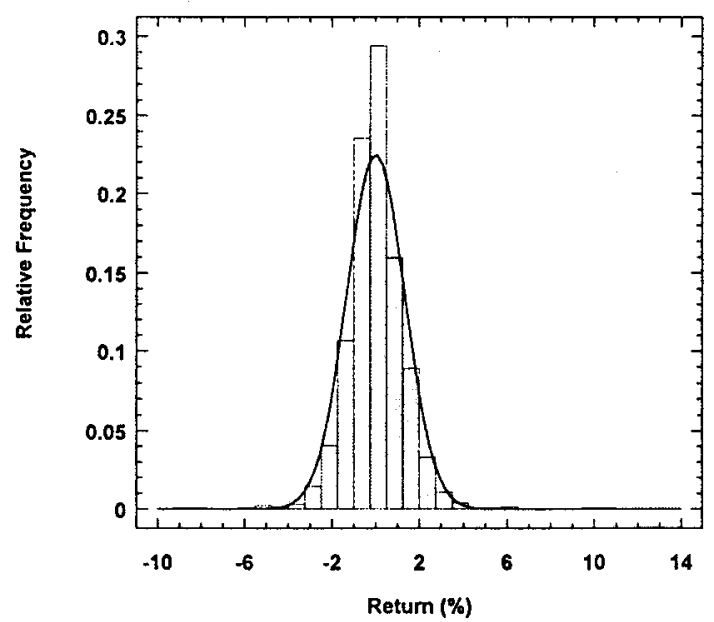



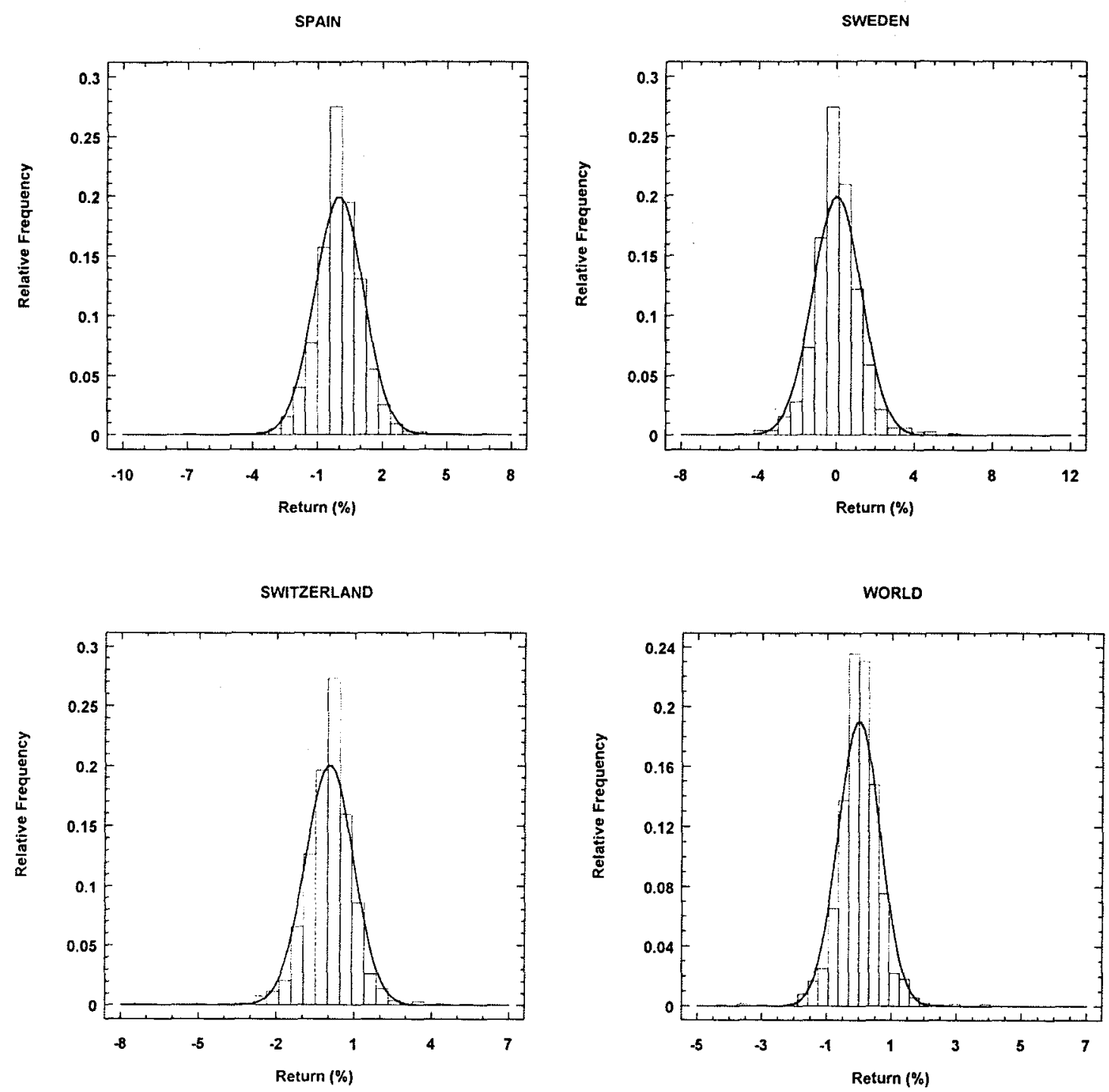


\section{REFERENCES}

Blattberg, R. and N. Gonedes (1974). "A Comparison of the Stable and Student Distributions as Statistical Models for Stock Prices." Journal of Business, 47, 244-280.

Bollerslev, T., R. Chou, and K. Kroner (1992). "ARCH Modeling in Finance: A Review of the Theory and Empirical Evidence." Journal of Econometrics, 52, 5-59.

Campbell, J., A. Lo, and C. MacKinlay (1997). The Econometrics of Financial Markets. Princeton University Press.

Clark, P. (1973). "A Subordinated Stochastic Process Model With Finite Variance for Speculative Prices." Econometrica, 41, 135-155.

Estrada, Javier (1997). "Random Walks and the Temporal Dimension of Risk." Carlos III University, Working Paper 97-24.

Fama, E. (1965). “The Behavior of Stock Market Prices.” Journal of Business, 38, 34-105.

Gray, B. and D. French (1990). "Empirical Comparisons of Distributional Models for Stock Index Returns." Journal of Business, Finance \& Accounting, 17, 451-459.

Hsu, D. (1982). "A Bayesian Robust Detection of Shift in the Risk Structure of Stock Market Returns." Journal of the American Statistical Association, March 1982, 29-39.

Kon, S. (1984). "Models of Stock Returns - A Comparison." Journal of Finance, 39, 147-65.

Mandelbrot, B. (1963). "The Variation of Certain Speculative Prices." Journal of Business, $36,394-419$.

Peiró, A. (1994). "The Distribution of Stock Returns: International Evidence." Applied Financial Economics, 4, 431-439.

Peters, E. (1991). Chaos and Order in the Capital Markets. A New View of Cycles, Prices, and Market Volatility. John Wiley and Sons.

Praetz, P. (1972). "The Distribution of Share Price Changes." Journal of Business, 45, 49. 55.

Press, J. (1967). “A Compound Events Model for Security Prices." Journal of Business, 40, 317-335.

Smith, J. (1981). "The Probability Distribution of Market Returns: A Logistic Hypothesis." Ph.D. dissertation, University of Utah. 